\title{
Erratum to: The effects of hematite nanoparticles on phytoavailability of arsenic and corn growth in contaminated soils
}

\author{
T. Mansouri ${ }^{1}$ - A. Golchin ${ }^{1}$ - M. R. Neyestani ${ }^{2}$
}

Published online: 13 March 2017

(C) Islamic Azad University (IAU) 2017

\section{Erratum to: Int. J. Environ. Sci. Technol. DOI 10.1007/s13762-017-1267-5}

Some text in Table 3 was missing. Below you can see the complete Table 3 .

The online version of the original article can be found under doi:10.1007/s13762-017-1267-5.

T. Mansouri

t.mansouri2010@gmail.com

1 Department of Soil Science, College of Agriculture,

University of Zanjan, Zanjan 4537138111, Iran

2 Novin Shimyar Laboratory, Tehran, Iran 
Table 3 Interactive effects of hematite nanoparticles application rates and levels of soil total As on the concentration of soil available As and concentrations of As (mean and standard error) in the root and aerial parts of the corn plant

\begin{tabular}{|c|c|c|c|c|}
\hline $\begin{array}{l}\text { Hematite nanoparticles } \\
\text { application rates }\end{array}$ & $\begin{array}{l}\text { Levels of soil total As } \\
(\mathrm{mg} / \mathrm{kg})\end{array}$ & $\begin{array}{l}\text { Concentration of available } \\
\text { As }(\mathrm{mg} / \mathrm{kg})\end{array}$ & $\begin{array}{l}\text { Concentration of As in } \\
\operatorname{root}(\mathrm{mg} / \mathrm{kg})\end{array}$ & $\begin{array}{l}\text { Concentration of As in aerial } \\
\text { parts }(\mathrm{mg} / \mathrm{kg})\end{array}$ \\
\hline \multirow[t]{6}{*}{0} & 0 & $0 \mathrm{o}$ & $0 \mathrm{p}$ & $0 \mathrm{~m}$ \\
\hline & 6 & $2.27 \pm 0.11 \mathrm{kl}$ & $12.861 \pm 0.29 \mathrm{~nm}$ & $6.838 \pm 0.09 \mathrm{i}$ \\
\hline & 12 & $3.64 \pm 0.07 \mathrm{j}$ & $20.703 \pm 0.281$ & $10.389 \pm 0.49 \mathrm{~h}$ \\
\hline & 24 & $6.908 \pm 0.16 \mathrm{~g}$ & $67.256 \pm 0.61 \mathrm{i}$ & $21.506 \pm 0.21 \mathrm{~g}$ \\
\hline & 48 & $14.683 \pm 0.26 \mathrm{c}$ & $197.29 \pm 1.27 \mathrm{~d}$ & $39.79 \pm 0.59 \mathrm{c}$ \\
\hline & 96 & $27.3 \pm 0.35 \mathrm{a}$ & $383.40 \pm 8.86 \mathrm{a}$ & $59.556 \pm 1.61 \mathrm{a}$ \\
\hline \multirow[t]{6}{*}{$0.05 \%$} & 0 & $0 \mathrm{o}$ & $0 \mathrm{p}$ & $0 \mathrm{~m}$ \\
\hline & 6 & $1.79 \pm 0.12 \mathrm{~lm}$ & $8.569 \pm 0.0 \mathrm{mno}$ & $3.505 \pm 0.05 \mathrm{kl}$ \\
\hline & 12 & $2.52 \pm 0.07 \mathrm{k}$ & $14.582 \pm 0.39 \mathrm{~lm}$ & $6.336 \pm 0.39 \mathrm{ij}$ \\
\hline & 24 & $5.34 \pm 0.06 \mathrm{~h}$ & $39.747 \pm 0.51 \mathrm{j}$ & $12.256 \pm 0.25 \mathrm{~h}$ \\
\hline & 48 & $10.07 \pm 0.09 \mathrm{e}$ & $131.823 \pm 1.30 \mathrm{f}$ & $27.608 \pm 1.22 \mathrm{e}$ \\
\hline & 96 & $18.38 \pm 0.24 \mathrm{~b}$ & $252.265 \pm 1.77 \mathrm{~b}$ & $44.000 \pm 0.85 \mathrm{~b}$ \\
\hline \multirow[t]{6}{*}{$0.1 \%$} & 0 & $0 \mathrm{o}$ & $0 \mathrm{p}$ & $0 \mathrm{~m}$ \\
\hline & 6 & $1.53 \pm 0.05 \mathrm{~nm}$ & $6.623 \pm 0.07$ nop & $2.748 \pm 0.23 \mathrm{kl}$ \\
\hline & 12 & $2.12 \pm 0.07 \mathrm{klm}$ & $12.194 \pm 0.22 \mathrm{mno}$ & $4.650 \pm 0.04 \mathrm{jk}$ \\
\hline & 24 & $4.26 \pm 0.05 \mathrm{i}$ & $31.314 \pm 0.99 \mathrm{k}$ & $11.501 \pm 0.03 \mathrm{~h}$ \\
\hline & 48 & $7.39 \pm 0.13 \mathrm{~g}$ & $107.533 \pm 0.24 \mathrm{~g}$ & $25.064 \pm 0.22 \mathrm{f}$ \\
\hline & 96 & $13.15 \pm 0.11 \mathrm{~d}$ & $217.58 \pm 1.16 \mathrm{c}$ & $38.234 \pm 0.75 \mathrm{c}$ \\
\hline \multirow[t]{6}{*}{$0.2 \%$} & 0 & $0 \mathrm{o}$ & $0 \mathrm{p}$ & $0 \mathrm{~m}$ \\
\hline & 6 & $1.14 \pm 0.04 \mathrm{n}$ & $4.764 \pm 0.14$ op & $2.273 \pm 0.081$ \\
\hline & 12 & $1.67 \pm 0.09 \mathrm{lmn}$ & $10.378 \pm 0.05 \mathrm{mno}$ & $4.062 \pm 0.22 \mathrm{kl}$ \\
\hline & 24 & $3.38 \pm 0.07 \mathrm{j}$ & $27.525 \pm 0.04 \mathrm{k}$ & $10.547 \pm 0.22 \mathrm{~h}$ \\
\hline & 48 & $5.82 \pm 0.34 \mathrm{~h}$ & $89.584 \pm 1.26 \mathrm{~h}$ & $21.028 \pm 0.82 \mathrm{~g}$ \\
\hline & 96 & $9.47 \pm 0.37 \mathrm{f}$ & $173.92 \pm 0.59 \mathrm{~d}$ & $35.62 \pm 0.26 \mathrm{~d}$ \\
\hline
\end{tabular}

The values shared a letter in common within a column are not significantly different $(p<0.01$, Duncan's multiple range test) 